\title{
A New Optimistic Mobility Model for Mobile Ad Hoc Networks
}

Tanweer Alam

Research Scholar, Department of Computer Sc \& Engg, Singhania University Pacheri Bari, Jhunjhnu

(Rajasthan) India

\author{
B.K. Sharma \\ PhD Guide, Senior Scientist, \\ N.I.T.R.A, Ghaziabad, India
}

\begin{abstract}
To produce a real-world environment within which an ad hoc network can be formed among a set of nodes, there is a need for the development of realistic, generic and comprehensive mobility models. Simulation environment is an important tool for the evaluation of new concepts in networking. Here, we show that the proposed mobility model has a significant impact on network performance, especially when compared to other mobility models. The mobile ad hoc networks depend on understanding protocols from simulations, before these protocols are implemented in a real-world setting. In this paper, we present a new mobility model for mobile ad hoc network. We have developed a complete environment in which network protocols can be studied on the basis of numerous performance metrics and we also observe that the performance of ad hoc network protocols is affected when different mobility scenarios are utilized.
\end{abstract}

\section{Keywords}

Mobility Models, Mobile Ad Hoc Networks, Simulation Area, Network Protocols, Real World environment.

\section{INTRODUCTION}

Mobility models are represented by the movement of mobile users, and they change their location, velocity and acceleration over time. These models are used for simulation purposes. For mobility modeling, the activity of a movement of user can be described using both analytical and simulation models [1].

When evaluating mobility models for wireless ad hoc networks with respect to performance or functional correctness, several assumptions have to be decided upon. Such assumptions may include the size and shape of the area used by the wireless devices, their transmission ranges and their movement patterns including allowed directional changes and speeds [2].

\section{AD HOC NETWORKS}

Ad Hoc is often used to describe solutions that are developed onthe-fly for a specific purpose. An ad hoc network refers to a network connection established for a single session and does not require a wireless base station such as router. Basically, it is a temporary network connection created for a specific purpose (such as transferring data from one computer to another) [3].

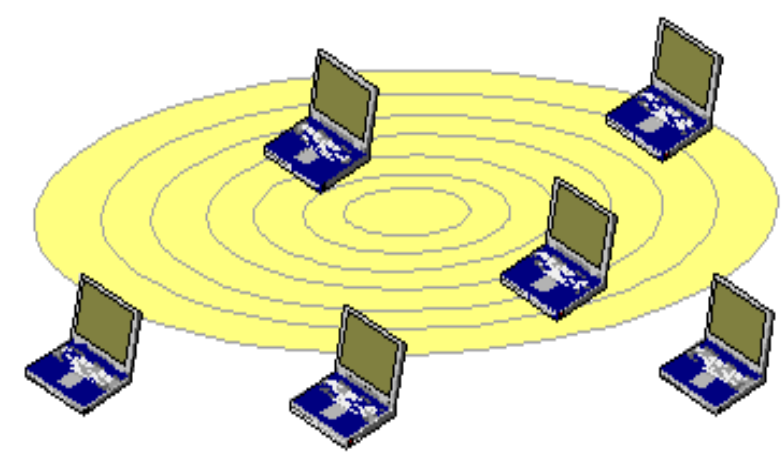

Fig. 1: Ad Hoc Client to Client

Infrastructure based networks pass information through a central information hub which can be a hardware device or software on a computer. An ad hoc network is infrastructure less network that is one where there are no access points passing information between participants. It can be thought of as a peer-to-peer network for the wireless age. Peer-to-peer or workgroup style networks were used to create a network environment for early Windows computers. This allowed these early computers to connect to each other to exchange information, usually in a smaller office environment without the need for domains and the additional management [4].

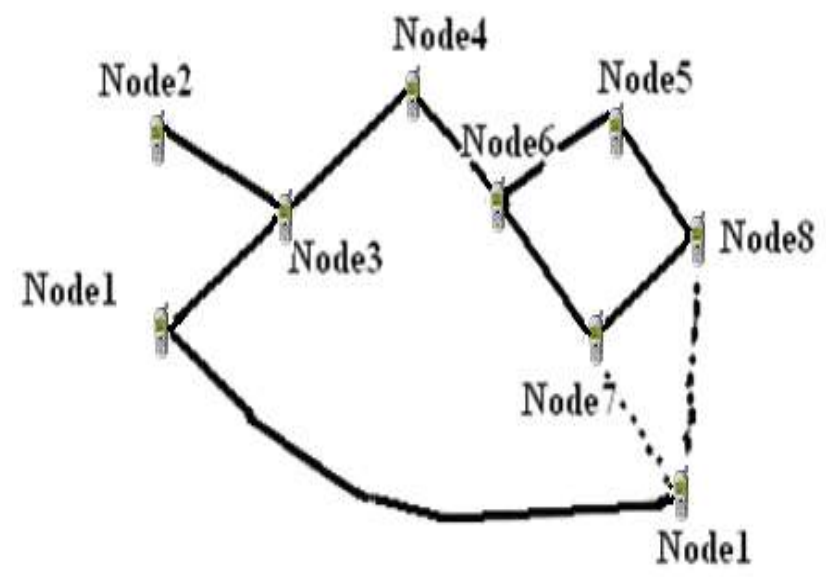

Fig. 2: Movement in Ad Hoc Network 
An ad-hoc network is a local area network that is built spontaneously as devices connect. Instead of relying on a base station to coordinate the flow of messages to each node in the network, the individual network nodes forward packets to and from each other [5].

\section{TRAVELING PATTERN OF MOBILE NODES}

The Random Waypoint Mobility Model is a widely used mobility model. The movement pattern of an MN using the Random Waypoint Mobility Model is similar to the Random Walk Mobility Model if pause time is zero and [minspeed, maxspeed] $=\left[\right.$ speed $_{\min }$, speed $\left._{\max }\right]$. It includes pause times between changes in direction or speed. A Mobile Node begins by staying in one location for a certain period of time. In the performance evaluation of a protocol for an ad hoc network, the protocol should be tested under realistic conditions including, but not limited to, a sensible transmission range, limited buffer space for storage of messages, representative data traffic models, and realistic movement of mobile users.

\section{EXISTING MOBILITY MODELS}

Mobility models play an important role in the evaluation of wireless network protocols. Ad hoc mobility models differ from cellular mobility models in the network. The connectivity and capacity of a network often depends on the nodes mobility behavior. Cellular mobility models require the use of BSs whereas ad hoc mobility models require the cooperation of two or more communicating MNs. Although separate mobility models exist for cellular and ad hoc mobility models, similarities exist between the two categories. The Random Waypoint model is one of the widely used models for ad-hoc and infrastructure wireless simulations and has been implemented in many network simulators. The following ad hoc mobility models are described in this section:

\subsection{Random Mobility Model}

Random Mobility Model is a simple mobility model based on random directions and speeds. The Random Walk Mobility Model was first developed mathematically by Einstein in 1926 [6]. In this mobility model a mobile node moves from its current location to a new location by randomly choosing a direction and speed in which to travel. The new speed and direction are both chosen from predefined ranges, $\left[\operatorname{speed}_{\min }, \operatorname{speed}_{\max }\right]$ and $[0,2 л]$ respectively.

\subsection{Random Direction Mobility Model}

Random Direction mobility Model is a model that forces Mobile Nodes to travel to the edge of the simulation area before changing direction and speed.

\subsection{A Boundless Simulation Area Mobility}

\section{Model}

A Boundless simulation area mobility model is a model that converts a $2 \mathrm{D}$ rectangular simulation area into a torus-shaped simulation area.

\subsection{Random Waypoint Mobility Model}

It is a model that includes pause times between changes in destination and speed. In this model nodes choose a random location on the simulation area and walk there with the speed drawn from a uniform distribution with a minimum and maximum value. When the node has arrived, it will wait a random amount of time, and then continue with the same routine.

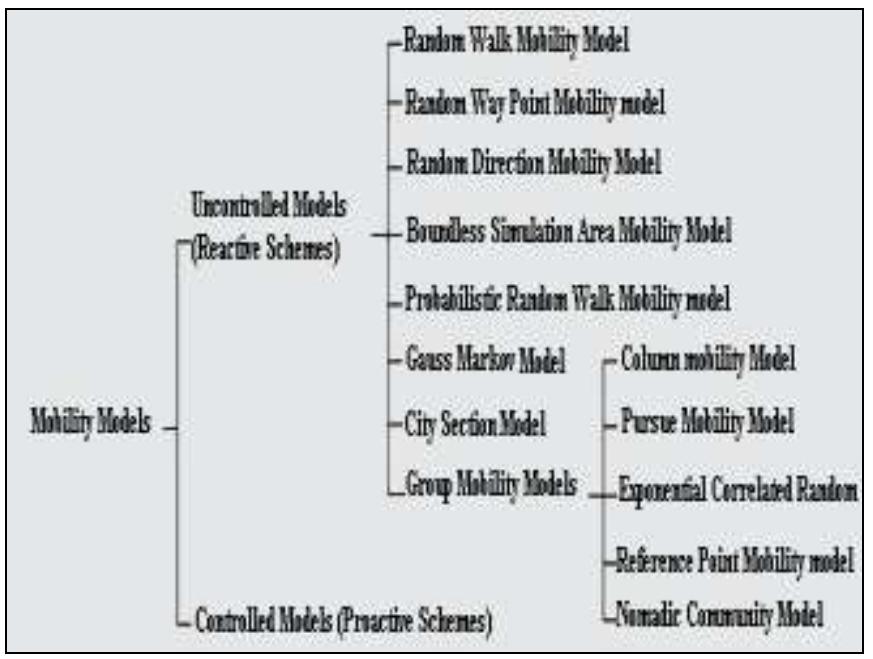

Fig.3: Category of Mobility Models

\subsection{A Probabilistic Version of the Random Mobility Model}

It is a model that utilizes a probability matrix to determine the next position of an $\mathrm{MN}$

\subsection{Constant Velocity Random Direction Mobility Model}

It is a revised version of the Random Mobility Model.

\subsection{City Area, Area Zone, and Street Unit Mobility Models}

These models describing simulation areas representing different granular scales of a city.

\subsection{A Hierarchy of Mobility Models}

A hierarchy of mobility models to represent varying degrees of scale for long distance travel.

In this paper we describe several mobility models that represent mobile nodes whose movements are independent of each other. In group mobility models that represent mobile nodes whose movements are dependent on each other [7]. The main goal of 
this paper is to present a new mobility model for mobile ad hoc network. We have developed a complete environment in which network protocols can be studied on the basis of numerous performance metrics and we also observe that the performance of ad hoc network protocols is affected when different mobility scenarios are utilized.

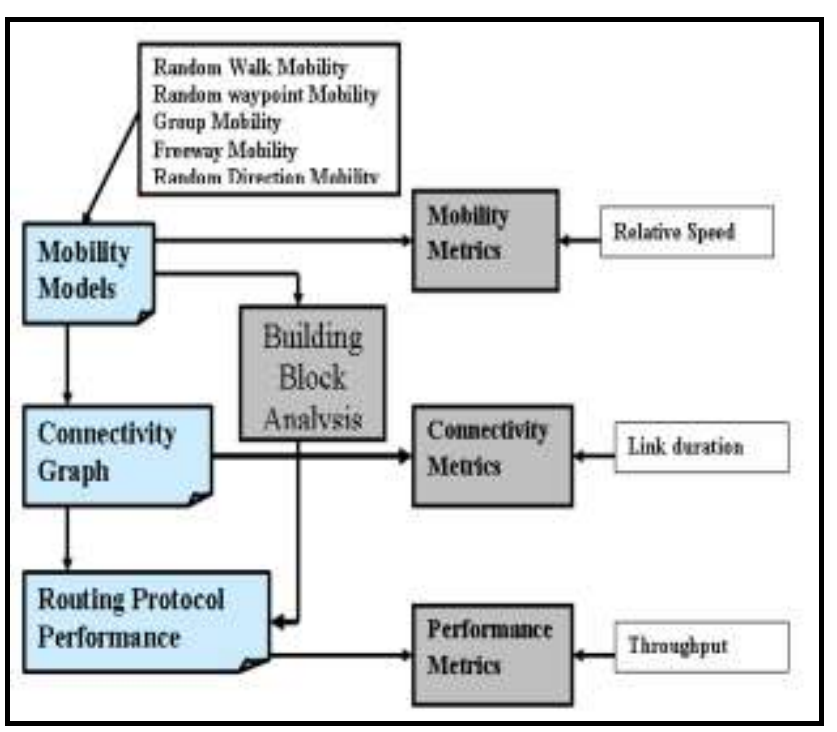

Fig.4: Framework of Mobility Model

\section{PROPOSED NEW MOBILITY MODEL}

The Obstacle Mobility Model is based on the following real-life observations. First, people move towards specific destinations rather than randomly choosing some destinations. Second, there are obstacles in the real world. These obstacles, most commonly the buildings block people's movements as well hinder signalpropagation. Third, people do not walk along random trajectories; they usually move along pathways and select shortest paths.

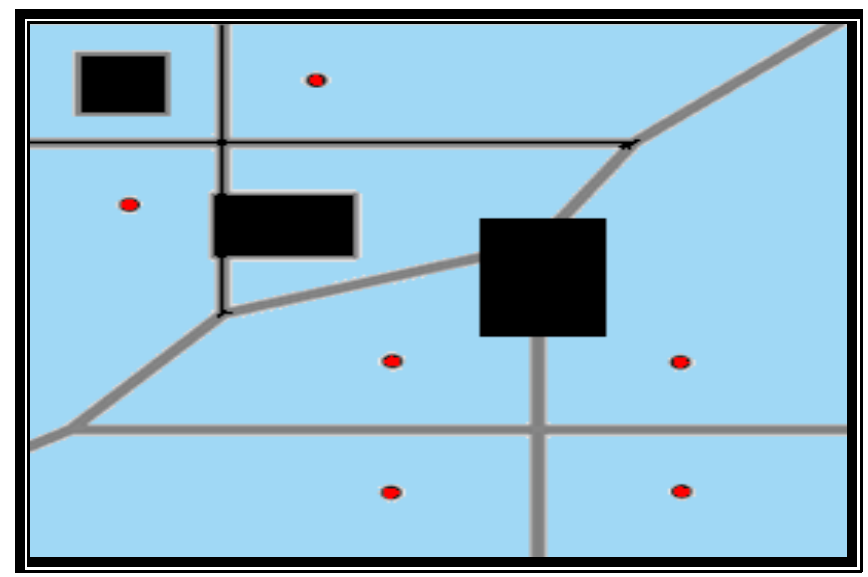

Fig.5: Example for Obstacle Mobility Model

The developed new obstacle mobility model does not rely on a graph. To move from a source location to a destination one, each node has to find its appropriate pathway through the environment. In order to allow nodes not to pass through obstacles, we implemented a path finding algorithm. This algorithm uses a ray launching technique coupled with an optimized sweep line algorithm for the fast ray intersection search computation.

\section{THE PATH FINDING ALGORITHM}

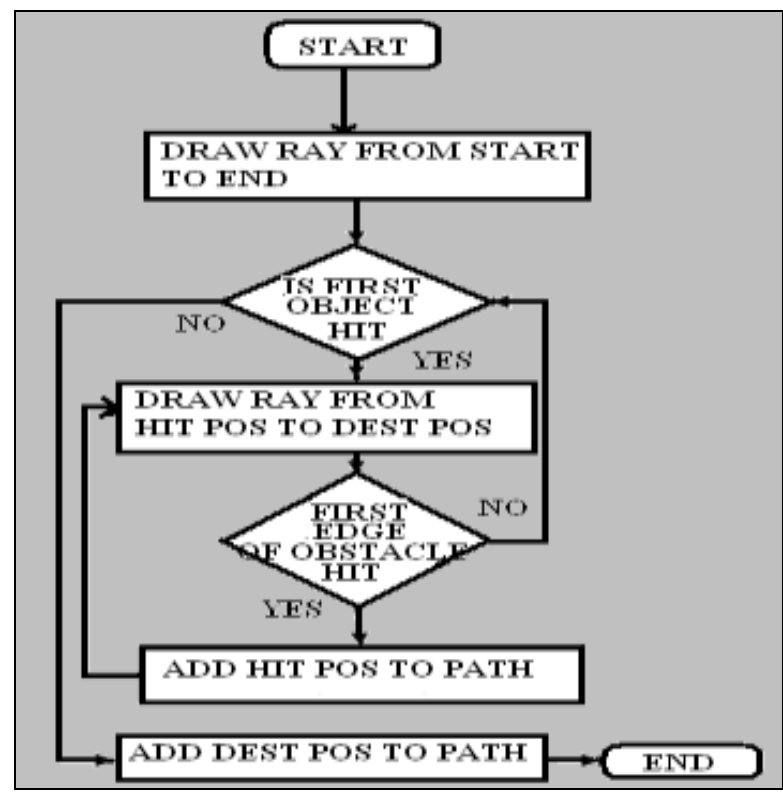

Fig.6: Path finding process flow

A path is a set of location points which form adjacent segments and no segment intersects with an obstacle in the environment. The algorithm is as follows.

Step 1: Initialize the starting and ending point.

Step 2: Draw ray between these two points.

Step 3: is first object hit? If yes go to step 4, otherwise go to step 7.

Step 4: draw ray from hit position to destination position.

Step 5: Now check first edge of obstacle hit. If it is then go to step 6 otherwise go to step 3 .

Step 6: add hit position to path. Go to step 4.

Step 7: add destination position to path. Go to step 8 .

Step 8: stop

At the beginning, the start position and the actual position are the same. We lunch a ray from source to destination and search for the first obstacle hit by this ray. Now we add the first hit point to the path and try to border this first obstacle. For do this, we search for the first edge hit in this obstacle. If an edge is hit, the actual position moves to the intersection point on this edge. We choose the nearest side of the hit edge in order to minimize the final path length. We repeat until the obstacle is encountered. This means that the ray from the node position to destination does not hit any edge of this obstacle [8]. 


\section{OPTIMIZATION OF THE MODEL}

For computing the fast intersection search of a ray with obstacles, we used a sweep line algorithm. This algorithm has a complexity of $n \log (n+k)$ with $k$ being the number of intersections and $n$ the number of vertices of all obstacles. The procedure is as follows.

i) First of all, a status structure stores the list of events such as add/remove a line segment from the list.

ii) A priority queue stores the sequence of line segments in the environment is maintained. The line segments are stored in an order.

iii) To compare two points we first examine the $\mathrm{X}$ coordinate, then the $\mathrm{Y}$ coordinate.

iv) To speed up this algorithm, the real time environment is clipped to a small rectangular zone each time we search a pathway for a node movement.

v) We choose the sub region between the node position and the destination. The path finding algorithm is then done only in this sub region because the obstacles out of this selected region are far away and will never be hit by a ray.

vi) A second performed optimization is to store in a list, the index of the first line segment situated on the left of each point coordinate.

vii) Without this information, the algorithm has to restart the sweep process at the first index in the priority queue each time its searches for an intersection.

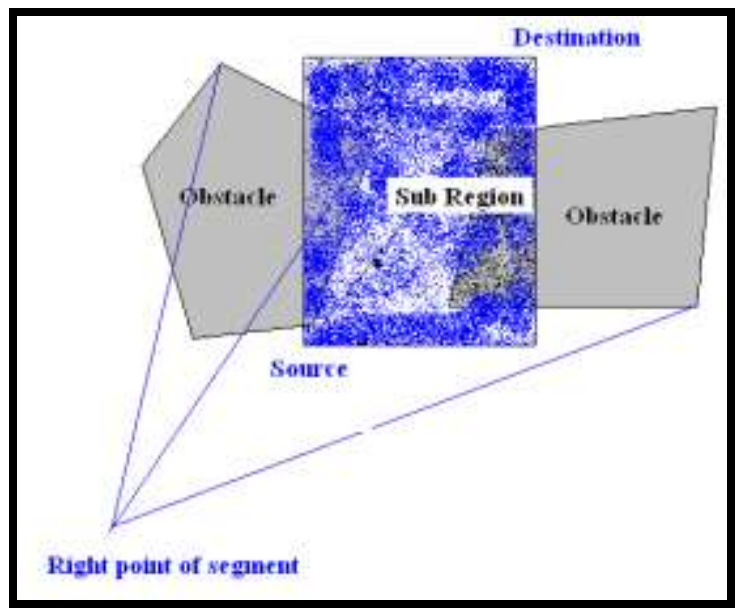

Fig.7: Sweep line intersection

\section{CONCLUSION}

Mobility models play an important role in the evaluation of wireless network protocols. Wireless ad hoc simulation models need to approximate accurately common real world environments in order for the simulations to assess conveniently any studied ad hoc communication protocol. The work presented in this paper introduced a new tool for facilitating the generation of realistic mobility models that include obstacles for ad hoc mobile networks. We implemented a path finding algorithm. This algorithm uses a ray launching technique coupled with an optimized sweep line algorithm for the fast ray intersection search computation. We can summarize this algorithm as follows. When a node needs to move from its location to a chosen destination location, a ray is launched from its actual position towards the destination position. We repeat this operation each time we want to find the next position to be added to a mobile node pathway towards its destination location.

\section{ACKNOWLEDGEMENTS}

My thanks to the experts who have contributed towards research in Mobility Models and ad hoc wireless networks.

\section{REFERENCES}

[1] wikipedia, "Mobility Models",

http://en.wikipedia.org/wiki/Mobility_model

[2] Tracy Camp, Jeff Boleng, and Vanessa Davies. A survey of mobility models for ad hoc network research. Wireless Communications and Mobile Computing, 2(5), 2002, Page no: 483-502.

[3]Techterms, "Ad Hoc Networks", http://www.techterms.com/definition/adhocnetwork

[4]wisegreek, "What is an ad hoc networks", http://www.wisegeek.com/what-is-an-ad-hoc-network.htm

[5] Searchmobilecomputing, Adhoc networks definition, http://searchmobilecomputing.techtarget.com/definition/ad-hocnetwork

[6] M. Sanchez and P. Manzoni. Anejos: A java based simulator for ad-hoc networks. Future Generation ComputerSystems, 17(5): pages 573-583, 2001.

[7]http://www.jr.ietejournals.org/article.asp?issn=03772063 ; year $=2007$; volume $=53 ;$ issue $=1$; spage $=3$; epage $=12 ;$ aulast $=\mathrm{R}$ adha;type $=0$

[8] http://www.gel.usherb.ca/interlab/downloads/amadeos.html

[9] V. Davies, T. Camp, and J. Boleng "A Survey of Mobility Models for Ad Hoc Network ",Special issue on Mobile Ad Hoc Networking, April 2002.

[10] I. Stepanov, "A framework for user mobility modeling" Project page available: http://canu.informatik.uni-stuttgart.de

[11] C. Bettstetter, "Smooth is Better than Sharp: A random Mobility Model for Simulation of Wireless Networks ", Proceedings of the 4th ACM International Workshop on Modeling, Analysis, and Simulation of Wireless and Mobile Systems (MSWIM), Rome, Italy, July 2001. 